\title{
Scientific uncertainty
}

\author{
The broad-scale impacts and mechanisms of physical climate change are scientifically well-understood, but \\ specific estimates of these impacts are associated with uncertainty that is challenging to communicate. \\ How scientists communicate uncertainty affects public trust and acceptance of the research.
}

\section{| /}

isten to the scientists" - some form of this phrase is often used in debates over the realities of climate change, including recently by climate activist Greta Thunberg in her testimony to the US Congress and by the participants in ongoing climate protests. Such phrases underline the scientific consensus that climate change is real and caused by anthropogenic greenhouse gas emissions ( $97 \%$ of climate scientists agree with these two points ${ }^{1}$ ) and convey the idea that science will pave the way forward by informing policy meant to limit greenhouse gas emissions and deal with climate change impacts.

The potential effects of elevated $\mathrm{CO}_{2}$ on the Earth's temperature were calculated as early as the late nineteenth century by Svante Arrhenius, using measurements of infrared radiation from the Moon ${ }^{2}$. Despite today's much more sophisticated computing power and deeper understanding of the Earth's climate system, these calculations have not been substantially revised. Since the subsequent realization that anthropogenic $\mathrm{CO}_{2}$ could affect Earth's climate, the evidence of the effects of increasing atmospheric levels of greenhouse gases has become clearer and more convincing. Data collected over the past three decades, in particular, have allowed scientists to test hypotheses and models as the effects of anthropogenic climate change have intensified and become easier to distinguish from natural variability. Temperatures are increasing and will continue to do so, ice caps and glaciers will continue to melt, and sea level will rise. This is the science, laid out most recently and with relative certainty by the IPCC Special Report on the ocean and cryosphere ${ }^{3}$.

However, the climate system is complex, as are its interactions with humanity, and beyond these 'big picture' forecasts lie many more specific questions. How much will sea level rise, and how quickly? How will forests respond to increased $\mathrm{CO}_{2}$ and changing precipitation patterns? Will life be able to adapt to the changing conditions? Research into the nuances of these and similar questions sometimes yields unclear or conflicting results. The answers may vary geographically and be confounded

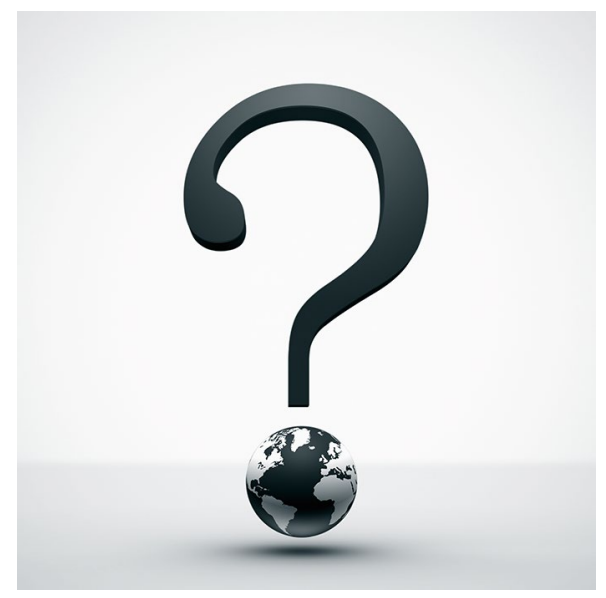

Credit: Jorg Greuel/DigitalVision/Getty

by natural variability, so that attribution of certain events to climate change is difficult. Future answers to these questions are typically calculated from models based on mathematical representation of processes or statistical regression of past data, both of which have limitations. Predictions are also uncertain because human and societal actions are unknown.

All of this makes precise predictions of impacts and timelines challenging. Yet, exactly these are highly relevant for informing mitigation policies and deciding how to react to the varied consequences of climate change. Governments need to know what will happen to decide how best to invest money and infrastructure; and they need to know what effect policies meant to reduce emissions and slow climate change will have. As more and more countries pass national and international legislation aimed to mitigate climate change, measuring the efficacy of these policies will become a priority that is likely to play a role in their strength and success, but one that may prove difficult because of discrepancies between political timescales and those required to resolve trends ${ }^{4}$.

The scientific uncertainty surrounding climate change research can be difficult to communicate to policy makers and the public ${ }^{5}$. The word "uncertainty" itself has slightly different meanings when used in everyday speech versus a scientific context.
In scientific discourse, it conveys the degree to which something is known. In the vernacular, the word conveys rather the sense of not knowing. The difference is subtle, but important.

An Article by Lauren Howe and colleagues in this issue reports that how scientists express this uncertainty affects people's acceptance of the science, as well as their trust in the scientists reporting the results. They find that expressing uncertainty using a concrete range of possibilities increases trust, whereas uncertainty expressed with respect to unpredictable impacts can erode trust in scientists and in the research. As Emily Ho and David Budescu discuss in the accompanying News \& Views, this may be because the public opinion of climate change is dependent on how the evidence is framed. Expressing 'irreducible' uncertainty may convey the idea that scientists disagree with each other, which lessens public confidence, whereas most people accept that it is not possible to predict the future precisely and are therefore amenable to ranges of potential outcomes.

As research progresses, the uncertainty surrounding impact estimates will probably continue to decrease. Researchers are quickly filling in knowledge gaps, improving models and grounding them in observations, all of which improve estimates and predictions. The scale and interconnectedness of the Earth/climate system, as well as the very nature of scientific research, mean that uncertainty will remain an integral part of the discourse, particularly with respect to future perspectives. Nevertheless, enough is known, and has been known for the past 100 years, to justify efforts to reduce emissions.

Published online: 29 October 2019 https://doi.org/10.1038/s41558-019-0627-1

References

1. Cook, J. et al. Environ. Res. Lett. 11, 048002 (2016).

2. Arrhenius, S. Lond. Edinb. Dublin Phil. Mag. J. Sci. 41, 237-276 (1896).

3. IPCC. Summary for Policymakers. IPCC Special Report on the Ocean and Cryosphere in a Changing Climate (eds. Pörtner, H.-O. et al.) (IPCC, in the press).

4. Marotzke, J. WIRES Clim. Change. https://doi.org/10.1002/ wcc.563 (2019).

5. Joslyn, S. L. \& LeClerc, J. E. Top. Cogn. Sci. 8, 222-241 (2016). 